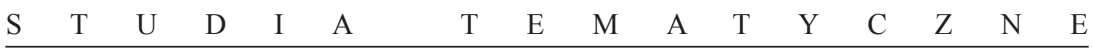

\title{
GOUVERNANCE DE LA RELIGION ET LIBERTÉ DE CONSCIENCE
}

Aux termes de l'article 18 de la déclaration des droits de l'homme et du citoyen: „toute personne a droit à la liberté de pensée, de conscience et de religion, ce droit implique la liberté de changer de religion ou de conviction ainsi que la liberté de manifester sa religion ou sa conviction seule ou en commun, tant en public qu'en privé, par l'enseignement, les pratiques, le culte et l'accomplissement des rites". La liberté de conscience apparait ainsi comme englobant la liberté de religion.

L'objet de cet étude sera de rechercher en quoi la liberté de conscience comme droit civil peut être déterminée par la façon dont la religion encadre la liberté de conscience à l'externe et en quoi la liberté de conscience comme droit politique pourrait être déterminée par la conception religieuse de la liberté de conscience à l'interne. Avant de s'intéresser à la parenté entre liberté de conscience et religion en droit, encore faut-il cerner le contenu de la liberté de conscience. Le rapport entre liberté de conscience et de religion ne peut être analysé qu'une fois définie la distinction entre la liberté de conscience et les autres libertés qui la jouxte.

${ }^{*}$ Dr, Science po Aix, Institut d'Études Politiques d'Aix-en-Provence, 25, rue Gaston de Saporta, 13625 Aix-en-Provence cedex 1, France, e-mail: clamarmar@yahoo.fr. 
La liberté de conscience implique la possibilité pour chacun de choisir les valeurs sur lesquelles il fonde son existence au sens strict, la liberté de conscience est une liberté du „for intérieur”, elle s'apparente donc à la liberté de pensée et peut difficilement être limitée ${ }^{1}$, si ce n'est par l'utilisation, dans des cas extrêmes, des méthodes totalitaires d'endoctrinement. Cette liberté est protégée de façon absolue. Entendue dans un sens plus large, elle implique la capacité d'agir de façon libre et devient ainsi le fondement de toute liberté. Dans cette dernière acception, elle tolère une limitation pour préserver l'ordre public en particulier. Elle rejoint la liberté d'opinion, c'est-à-dire le droit pour chacun de ne pas être inquiété par son opinion et la liberté d'expression, droit pour chacun de manifester publiquement son opinion. Ses libertés se recoupent bien souvent. La personne religieuse peut percevoir l'expression de sa conscience comme faisant partie intégrante de son identité. Cette nécessité d'expression, quoiqu'elle puisse s'imposer à d'autres aspects de la liberté de conscience, apparaît particulièrement perceptible en matière de liberté de religion.

Les sources juridiques de la liberté de consciences contiennent le plus souvent des références à la religion ${ }^{2}$. L'article 10 de La Déclaration des droits de l'homme et du citoyen (DDHC) dispose que ,nul ne doit être inquiété pour ses opinions, même religieuses, pourvu que leur manifestation ne trouble pas l'ordre public établi par la Loi"'. L'expression „même religieuse” exprime la tension, clairement perçue dans le contexte de l'époque comme elle fut rédigée, entre religion et liberté de conscience. L'article $1^{\text {er }}$ de la loi de 1905 évoque également la tension entre liberté de conscience et religion lorsque, consacrant la liberté de conscience, il prévoit que la république ,garantit le libre exercice des cultes sous les seules restrictions édictées ci-après dans l'intérêt de l'ordre public". Enfin, c'est une décision relative au financement des établissements privés sous contrat qui fut l'occasion pour le conseil

${ }^{1}$ Hannah Arendt, Le système totalitaire. Les origines du totalitarisme (Paris: Points, 2005), 657.

${ }^{2}$ Le premier amendement de la constitution américaine préserve la liberté de conscience.

${ }^{3}$ On pourrait également citer, entre autres exemples, La Convention américaine relative aux droits de l'homme, adoptée à San José, Costa Rica, le 22 novembre 1969, à la Conférence spécialisée interaméricaine sur les Droits de l’Homme. 
constitutionnel de reconnaître que la liberté de conscience constituait un PFRLR 4 .

L'article 9 de la Convention de sauvegarde des Droits de l'Homme et des Libertés fondamentales $(\mathrm{CEDH})^{5}$, ansi que l'article 18 de la DUDH $^{6}$, consacrent à la fois la liberté de conscience et les libertés de pensée et de religion. Le Pacte international relatif aux droits civils et politiques des Nations Unies considère que la liberté de Conscience, comme de la liberté religieuse, constitue un droit indélogeable, "noyau intangible" des droits de l'homme.

L'article 14 interdit les discriminations, notamment celles fondées sur la religion, tandis que l'article 2 du premier protocole additionnel à la convention EDH prévoit le droit pour les parents d'assurer l'éducation et l'enseignement de leurs enfants conformément à leurs convictions religieuses.

La jurisprudence de la Cour européenne des Droits de l'Homme considère comme un élément essentiel au fonctionnement des sociétés démocratiques et considère que: „Telle que la protège l'article 9, la liberté de pensée, de conscience et de religion représente l'une des assises d'une « société démocratique » au sens de la Convention. Elle

${ }^{4}$ Décision $n^{\circ} 77-87$ DC du 23 novembre 1977. Journal officiel du 25 novembre 1977, page 5530, Recueil, p. 42.

ECLI:FR:CC:1977:77.87.DC. Le Conseil constitutionnel est décide (article premier: La loi complémentaire à la loi $n^{\circ} 59-1557$ du 31 décembre 1959 modifiée par la loi $n^{\circ}$ 71-400 du 1er juin 1971 et relative à la liberté de l'enseignement est déclarée conforme à la Constitution).

${ }^{5}$ Sur l'article 9 de la Convention de sauvegarde des Droits de 1'Homme et des Libertés fondamentales, voir Jim Murdoch, La protection du droit à la liberté de pensée, de conscience et de religion par la Convention européenne des droits de l'homme. Série des précis sur les droits de l'homme du Conseil de l'Europe (Strasbourg: Les ateliers du Conseil de l'Europe, 2012), 101. Son contenu est repris par l'article 10 de la Charte des droits fondamentaux de 1'Union européenne.

${ }^{6}$ Déclaration Universelle des Droits de l'Homme (DUDH) est adoptée par l'Assemblée générale des Nations unies le 10 décembre 1948 à Paris au palais de Chaillot par la résolution 217, art. 18: „Toute personne a droit à la liberté de pensée, de conscience et de religion; ce droit implique la liberté de changer de religion ou de conviction ainsi que la liberté de manifester sa religion ou sa conviction seule ou en commun, tant en public qu'en privé, par l'enseignement, les pratiques, le culte et l'accomplissement des rites". Cette définition a été complétée par la version de 1966. 
figure, dans sa dimension religieuse, parmi les éléments les plus essentiels de l'identité des croyants et de leur conception de la vie, mais elle est aussi un bien précieux pour les athées, les agnostiques, les sceptiques ou les indifférents. Il y va du pluralisme - chèrement conquis au cours des siècles - consubstantiel à pareille société"?.

Dans le cadre du référé ,liberté”, le Conseil d'État français a pour sa part, qualifié la liberté de culte de liberté fondamentale au sens de 1'article L. 521-2 du code de justice administrative ${ }^{8}$. Il évoque également ,un principe constitutionnel de liberté d'expression religieuse ${ }^{9 "}$. Néanmoins les systèmes juridiques nationaux ne consacrent pas systématiquement la liberté de culte, lui privilégiant parfois la notion de laïcité, comme c'est le cas du système français. La laïcité peut garantir la protection politique de la liberté de conscience. La liberté de conscience $^{10}$ est d'ailleurs définie comme ,second pilier de la laïcité” par la Commission de réflexion sur l'application du principe de laïcité dans la République française.

L'histoire a montré la capacité de pouvoirs religieux d'exiger une limitation de la liberté de conscience ${ }^{11}$. Cette limitation est justifiée par la source traditionnelle ${ }^{12}$ de la religion, s'opposant à la volonté générale, source du droit démocratique. La limitation de la liberté de conscience par la religion n'est pourtant pas systématique. Nombre de religions ont pu coexister pacifiquement ${ }^{13}$. L'histoire ne suffit donc pas à caractériser une opposition nécessaire entre religion et liberté de conscience.

${ }^{7}$ Arrêt de la Cour européenne des Droits de l’Homme, 25 mais 1993, affaire Kokkinakis c. Grèce (requête no 14307/88).

${ }^{8}$ Conseil d'Etat, du 16 février 2004, n ${ }^{\circ} 264314$.

${ }^{9}$ Conseil d'État, du 27 juin $2008, n^{\circ} 286798$.

${ }^{10}$ Sur l'expérience québécoise en matière de multiculturalisme: Gérard Bouchard, Charles Taylor, Fonder l'avenir. Le temps de la conciliation. Rapport de la Commission de consultation sur les pratiques d'accommodement reliées aux différences culturelles (Québec, 2008).

${ }^{11}$ La censure exercée sur Savonarole par le Pape Alexandre VI ou le massacre de la Saint-Barthélemy constituent des exemples de restrictions de la liberté de religion commis au nom de la religion.

${ }^{12}$ La source traditionnelle repose sur le caractère obligatoire de la règle coutumière.

${ }^{13}$ Mario Toscano, „Coexistence et histoire des religions”. Politique étrangère 20, no 5 (1955): 591-594. DOI: 10.3406/polit.1955.2564. 
Dans le monde contemporain, en particulier dans les démocraties occidentales, le débat portant sur la notion de liberté de conscience semble avoir d'ailleurs changé de lieu. Il ne porte plus tant sur ses violations les plus frappantes, discrimination ou même des personnes ne partageant pas la doctrine admise, que sur des entraves élimination plus discrètes telles que la limitation de l'expression religieuse ou de l'objection de conscience. Alors que le caractère inadmissible des premières violations citées fait désormais consensus dans le monde démocratique, le cœur véritable des secondes demeure toujours difficile à cerner.

Les liens entre liberté de conscience et religion peuvent être appréhendés de diverses façons. L'encadrement de l'expression de la religion, découlant de la liberté de conscience, en est un. Le problème de l'expression de signes religieux dans l'espace public, en particulier, a fait l'objet d'une littérature abondante en raison de ses conséquences pratiques $^{14}$. Cette question dépend largement des liens entre l'État et la religion: État laïque, confessionel, multi-confessionel ${ }^{15}$. Elle dépend également de la responsabilité de l'État envers les individus voir envers des communautés. La religion, et particulièrement les religions personnalistes, associent les dimensions personnelle et communautaire de l'identité. Par conséquent limiter la liberté religieuse produit à la fois des effets sur les institutions qui représentent une communauté religieuse et sur les personnes qui composent cette communauté. La limitation de cette liberté, comme celle d'autres droits de l'homme, pourrait impliquer une responsabilité de l'État envers les personnes concernées par cette limitation. Cette responsabilité ne peut être écartée au motif que dans la théorie politique française, la responsabilité publique ne porte de façon directe que sur les citoyens et non sur les communautés. En effet, elle est admise par les personnes croyantes et relève dès lors de leur liberté de conscience, que cette association entre identité personnelle

${ }^{14}$ Le débat français relatif au port de signes religieux dans les lieux public a montré que le port de tels signes pouvait être conçu dans certaines religions comme l'expression d'une identité personne. Loi n ${ }^{\circ} 2004-228$ du 15 mars 2004 encadrant, en application du principe de laïcité, le port de signes ou de tenues manifestant une appartenance religieuse dans les écoles, collèges et lycées publics, JORF n ${ }^{\circ} 65$ du 17 mars 2004 , page 5190 , texte $n^{\circ} 1$.

${ }^{15} \mathrm{Au}$ Liban par exemple, 18 religions sont reconnues par l'Etat. La place de l'islam et du christianisme dans l'État sont organisés par convention constitutionnelle. 
et communautaire soit fondée où qu'elle soit le résultat d'instrumentalisation spirituelle par les communautés pour leur propre subsistance.

Plutôt qu'à ces questions relatives à l'influence du politique sur la religion, nous nous intéresserons aux effets sur le droit de la pensée religieuse afin d'évaluer ses effets sur la reconnaissance juridique de la liberté de conscience en démocratie. Une méthodologie non juridique permet en effet l'analyse de phénomènes de gouvernance. Parmi la multitude d'aspect que revêt le concept de gouvernance ${ }^{16}$, nous nous intéresserons à l'influence de règle sur l'exercice du pouvoir ${ }^{17}$. Il s'agira de déterminer en quoi la reconnaissance juridique de la libre conscience pourrait être d'inspiration religieuse, autrement dit, de préciser le rôle de la religion dans l'élaboration d'un concept juridique. Il faudra en particulier établir si, sous couvert de religion, des logiques culturelles déterminaient le contenu de la liberté de conscience. L'influence religieuse sur la reconnaissance juridique de la liberté de conscience n'implique pas d'obligation pour les institutions de se référer à une telle source ${ }^{18}$.

Nous rechercherons si la religion implique une limitation de la liberté de conscience car c'est souvent en creux, lorsqu'il devient nécessaire de limiter la liberté de conscience pour permettre la coexistence de ces dernières, que se définit le cœur véritable de cette liberté.

Si la parenté entre la liberté de conscience et religion peut apparaître évidente, en particulier pour des croyants, elle n'en demeure pas moins

${ }^{16}$ Institut de Recherche et Débat sur la Gouvernance, http://www.institut-gouvernance. org.

${ }^{17} \mathrm{La}$ Commission européenne, dans le Livre blanc de la gouvernance européenne, définit la gouvernance comme les ,règles, les processus et les comportements qui influent sur l'exercice des pouvoirs au niveau européen".

Communication de la Commission, du 25 juillet 2001, „Gouvernance européenne - Un livre blanc" [COM(2001) 428 final - Journal officiel C 287 du 12.10.2001].

${ }^{18}$ Jean-François Gaudreault-Desbiens, „Religion, expression et libertés: l'offense comme raison faible de la régulation juridique", Cahiers de la Recherche sur les Droits Fondamentaux 8 (2010): 53-66. D'autre part, l'État n'est pas vu comme la source unique de droit. Au contraire, des ordres juridiques non étatiques, religieux ou autres, sont reconnus comme des sources de droit potentiellement signifiantes pour les citoyens, parfois autant sinon plus que les ordres juridiques étatiques. Capables de hiérarchiser et de moduler leurs appartenances juridiques diverses sans pour autant en occulter les limites, ces citoyens se retrouvent donc eux-mêmes à agir comme producteurs de droit, parfois à l'insu de l'État, occasionnellement en conflit avec celui-ci mais le plus souvent en coopération avec lui. 
quotidiennement controversée. Ce sujet, si dense, apparaît essentiel puisque la liberté de conscience produit des effets sur toutes les branches du droit, du droit civil au droit constitutionnel ${ }^{19}$. Qui plus est, une telle question n'est pas sans portée pratique ${ }^{20}$.

Il ne s'agira pas de procéder, de façon microscopique, à une étude de théologie comparée, d'histoire ou de sociologie des religions mais plutôt d'identifier, en quoi la religion, par principe, constitue une source d'influence dans la définition juridique à la liberté de conscience. Les pratiques, les sources ou la doctrine des différentes religions seront donc abordés à titre de preuve et non en tant qu'exposé synthétique. De plus, une simple comparaison entre la conscience en religion et la liberté de conscience ne saurait caractériser les liens entre conscience en religion et liberté de conscience. En effet, la notion de liberté de conscience titre ses fondements dans la philosophie antique ${ }^{21}$. Ainsi a-t-elle pu imprégner à la fois la religion ${ }^{22}$ et le monde politique moderne ${ }^{23}$. Par conséquent, une parenté théorique entre la liberté de conscience et la conception religieuse de la conscience ne suppose pas nécessairement que la première découle de la seconde. Ces deux notions pourraient qui plus est avoir subit une évolution commune.

${ }^{19}$ L'interprétation de la liberté de conscience détermine par exemple le contenu de la liberté de réunion, du droit du travail, du droit de la famille.

${ }^{20}$ Sur les liens entre religion et Etat au Luxembourg: „En matière religieuse et idéologique, l'État respecte, en vertu du principe de séparation, les principes de neutralité et d'impartialité. La loi règle les relations entre l'État et les communautés religieuses, ainsi que leur reconnaissance. Dans les limites et formes fixées par la loi, des conventions à approuver par la Chambre des Députés peuvent préciser les relations entre l'État et les communautés religieuses reconnues". Commission des Institutions et de la Révision constitutionnelle, Procès-verbal de la réunion du 21 janvier 2015. Voir Commission consultative des Droits de l'Homme du Grand-Duché de Luxembourg. L'Etat luxembourgeois et la laïcité.

${ }^{21}$ Sur la liberté dans la philosophie grecque: Aristote, René-Antoine Gauthier et Jean-Yves Jolif, Éthique à Nicomaque, Louvain-la-Neuve; Paris: Sterling (Virginia), Peeters (4 volumes), 2002, 2e éd. (1re éd. 1958-1959) (notice BnF no FRBNF31730090).

${ }_{22}$ Sur les liens entre le droit antique et actuel, voir Mogens Herman Hansen, Démocratie athénienne - Démocratie moderne: tradition et influences (Vandoeuvre-Genève: Entretiens sur 1'Antiquité Classique 56, 2010), 710. Ils s'agit là d'une exemple, une racine commune au principe de liberté de conscience et à la religion peut être observée dans d'autres cultures.

${ }^{23}$ Sur l'influence de la pensée grecque sur le christianisme: Aimé Puech, Histoire de la littérature grecque chrétienne depuis les origines jusqu'à la fin du IVeme siècle, 3 tome, (Paris: Société d'édition Les Belles Lettres, 1928-1930). 
Il apparaît utile, pour déterminer comment traiter le fait religieux en démocratie, de prendre en compte le point de vue religieux. Une telle approche montre le caractère limité d'une définition purement objective de la liberté de conscience. A l'inverse, une protection totalement subjective de la liberté de croyance et de conscience apparaît la porte ouverte à l'expression de tous les fondamentalismes, nécessitant les accommodements les plus laborieux. L'interdisciplinarité apparaît nécessaire à la conciliation de ces deux approches.

Nous rechercherons quelle influence exerce la conception religieuse de la liberté de conscience sur sa reconnaissance en démocratie. Cerner l'influence de la religion sur la liberté de conscience permet de déterminer dans quelle mesure la neutralité axiologique de l'État nécessite son encadrement du fait religieux lorsqu'il s'avère susceptible d'altérer la liberté de conscience.

Pour répondre à cette question, nous adopterons un point de vue externe et analyserons le phénomène religieux, de façon globale, comme un fait social. Il s'agira de préciser de façon qualitative, les liens entre l'encadrement par la religion de la liberté de conscience et la protection juridique de cette dernière en démocratie. La religion détermine la liberté de conscience à l'externe lorsqu'elle encadre le comportement de ses adeptes (en particulier le droit d'adopter une religion, de changer ou de quitter une religion) et à l'interne lorsqu'elle régule la contribution de chaque conscience dans la communauté à l'identification de vérité. En démocratie, la liberté de conscience, comme droit civil, constitue la garantie d'un pouvoir ,pour le peuple”; comme droit politique, elle assure l'expression d'un pouvoir ,du peuple par le peuple"24.

Nous étudierons en quoi la religion peut induire une restriction de la liberté de conscience (I) avant de rechercher si cette restriction est une nécessité découlant du phénomène religieux même (II).

${ }^{24}$ La liberté de conscience en tant que droit civil a pour sujet chaque citoyen, pour autant, comme droit culturel, son objet et social. 


\section{LA POSSIBLE RESTRICTION DE LA LIBERTÉ DE CONSCIENCE PAR LA RELIGION}

L'identification de dogmes religieux par les autorités religieuses n'est pas conçue comme reposant sur l'expression libre des consciences (A). Ce faisant, la religion a souvent défendu la nécessité de limiter la liberté de conscience comme droit civil (B).

\section{A. LA SOURCE TRADITIONNELLE DE LA RELIGION COMME CAUSE POTENTIEL- LE DE RESTRICTION DE LA LIBERTÉ DE CONSCIENCE}

La religion repose sur une source traditionnelle. La religion tolère un encadrement de la liberté de conscience en son sein lorsque la délibération vise à découvrir une vérité. Cette limitation est justifiée par le processus de découverte ${ }^{25}$ utilisé par la religion ${ }^{26}$.

En effet, il est communément admis que la religion, proposant un principe hétéronome (c'est-à-dire qu'elle propose une régulation de la société par des règles qui lui sont extérieures), comme principe de base de la société, reposerait sur une source traditionnelle. La délibération, quoique strictement encadré, fait partie intégrante de la source traditionnelle. Il s'agit de déterminer si le concept même de religion implique un tel fondement, ce qui le rendrait incompatible avec le régime démocratique:

„La théocratie et la démocratie apparaissent donc inconciliables comme le sont la toute-puissance arbitraire et le dissentiment qui s'y oppose avant de la combattre parfois jusqu'à la mort. Par suite, si la pensée juive se trouve conglomérée à l'idée d'un Dieu théocratique, il faudrait

${ }^{25}$ Nous désignerons par cette expression les procédés utilisés par les instances religieuses pour dégager des dogmes religieux. Par opposition au processus de justification, le processus de découverte ne découle pas nécessairement d'un raisonnement logique. Il se borne à observer un lien entre des phénomènes sans l'expliquer de façon rationnelle.

${ }^{26}$ A titre d'exemple: Charles Morerod, „Dogme et Oecuménisme”, Nova et Vetera 78/1-2 (2003): 29-61; Claude Tresmontant, Introduction à la théologie chrétienne (Paris: Seuil, 1974). 
besogner dur pour tenter de démontrer qu'il y a bien compatibilité entre elle et le concept de liberté" 27 .

La religion considère nécessairement la vérité comme d'origine divine et non définie par l'homme. Le sens social n'est pas auto-institué mais transcendant ${ }^{28}$ et le social est institué par un principe exogène ${ }^{29}$, ce qui suppose l'existence d'une communauté de conviction. La vérité, en religion, est intuitive davantage que discursive ${ }^{30}$. Elle est accessible à la foi, de façon ,arationnelle”. En effet, l'épistémologie ${ }^{31}$ religieuse ne repose en général pas sur un modèle purement déductif. La réflexion prend un texte, inspiré ou sacré, pour prémisse. La validation d'une interprétation de ce texte est déterminée par des témoignages, lesquels contribuent à la constitution de la tradition.

Le dialogue religieux se trouvant limité par la prétention de chaque religion à rendre compte de la vérité de façon singulière et intégrale ${ }^{32}$, l'œcuménisme montre le caractère intuitif de la découverte de la vérité religieuse. Entre autres exemples, l'Église catholique, produisant des effets de sens d'ordre épistémologique et politique, pourrait ainsi avoir induit une conception jusnaturaliste du monde ${ }^{33}$. La doctrine sociale de

${ }^{27}$ Raphaël Draï, „La liberté dans la pensée juive”, Raisons politiques 4/8 (2002): 61-80. DOI 10.3917/rai.008.0061. Raphaël Draï, La Thora. La législation de Dieu (Paris: Michalon, 2000).

${ }^{28}$ Jean-Marc Ferry, Conférence sur le thème: „Agnosticisme constitutionnel et foi religieuse dans l'espace européen, La raison publique agnostique peut-elle accueillir en son sein les expressions de l'expérience religieuse?”, donnée au Séminaire „Raison publique, éthique et foi religieuse dans 1'espace public européen”, du Professeur Denis Müller de l'Université de Lausanne (Suisse) le 27 juin 2008, Faculté de Théologie.

${ }^{29}$ C'est-à-dire, dans ce contexte provenant de l'extérieur de la société.

${ }^{30} \mathrm{C}$ 'est à dire qu'elle est accessible à 1 connaissance de façon directe et non par le discours.

${ }^{31}$ Nous emploierons le terme épistémologie dans son acception la plus large, comme désignant la théorie de la connaissance.

${ }^{32}$ Ainsi catholique signifie ,universel”. Voir aussi les actes du colloque organisé par le Centre d'études et d'interprétation du fait religieux (CEDIFR) le 27 et 28 février 2014, „Religions entre universalité et pluralisme”.

${ }_{33}$ Ainsi, l'explication du politique relèverait-elle davantage de la métaphysique et de la théologie que de la sociologie ou de l'histoire. Hannah Arendt, Essays in Understanding: 1930-1954. Formation, Exile, and Totalitarianism, edited by Jerome Kohn, (New York: 
l'Église catholique, sans se prononcer sur la relation ontologique entre droit positif et droit naturel, pose la soumission du droit positif au droit religieux comme obligation morale, ce qui a pu amener une partie de la doctrine juridique à considérer le lien entre droit positif et naturel comme nécessaire.

En raison, notamment, du processus de découverte employé par la religion, certains arguments religieux apparaissent difficilement traductibles en des termes purement séculiers. Si elle n'est pas hermétique, une frontière demeure entre les objets respectifs de la délibération publique et la délibération religieuse ${ }^{34}$. La délibération publique, entendu comme procédé permettant aux institutions politiques publiques la recherche de l'intérêt général ${ }^{35}$, doit pour produire une norme démocratique, satisfaire certaines conditions de représentativité ${ }^{36}$. Dans la théorie habermassienne, la délibération publique apparaît comme le fondement légitime de toute décision politique. Ce processus discursif idéal permet aux justiciables de répondre collectivement à un problème d'intérêt général par une action communicative réciproque. Pour ce faire, la délibération publique doit être soumise à diverses conditions telles que l'universalité, l'inclusion, l'entente, la rationalité et l'efficacité politique. De telles exigences ne s'imposent pas à la délibération entre autorités religieuses ${ }^{37}$.

Néanmoins, cette frontière n'exclue pas la gouvernance de la religion sur la reconnaissance de la liberté de conscience. En effet, la valeur subjective de convictions religieuses peut être admise par la sphère publique. Ainsi de nombreuses normes autorisant la libre pratique d'usages religieux ou le libre port de signes religieux se trouvent-elle

Harcourt Brace\&Co., 1994). Texte traduit par Etienne Tassin et publié dans Les Origines du totalitarisme. Eichmann à Jérusalem (Paris: Collection Quarto, Gallimard, 2002), 967-974.

${ }^{34}$ Par délibération religieuse, nous désignons l'ensemble des processus dialectiques utilisé par des institutions religieuses pour définir les canons d'une religion

${ }^{35}$ Jean-Noël Ferrié, Baudouin Dupret, Vincent Legrand, „Comprendre la délibération parlementaire. Une approche praxéologique de la politique en action”, Revue française de science politique 58, n 5 (2008): 795-815.

${ }^{36}$ Jürgen Habermas, Théorie de l'agir communicationnel, tomes 1, (Paris: Fayard, 1981), 448.

${ }^{37}$ Voir Léo Moulin, „Les origines religieuses des techniques électorales et délibératives modernes". Politix. Revue des sciences sociales du politique 43 (1998): 117-162. 
justifiées $^{38}$. Cette dernière, au titre de la liberté de croyance, pourrait tolérer de telles convictions sans les admettre.

Il semblerait que l'universalisme religieux ${ }^{39}$ ne se justifie pas tant par des motifs théologiques que par des motifs anthropologiques. La tendance de la religion à se transmettre d'une personne à un groupe davantage que de façon interpersonnelle peut entraver la transmission par son biais d'une pensée libérale encourageant l'autonomie de chacun des membres de la sociétét ${ }^{40}$. La religion assure dans un groupe une fonction normative. En cela, elle peut apparaître nécessaire à sa pérennitét ${ }^{41}$.

Aussi, la limitation de la liberté de conscience pourrait découler non seulement d'une interprétation erronée de la religion mais aussi de son instrumentalisation comme vecteur identitaire, résultant d'un défaut de „conscience politique"42. La délibération serait limitée afin de préserver une culture, un faisceau de valeurs localisé. Il ne s'agirait pas d'accéder à une Vérité universelle, mais à une vérité conjoncturelle. Par conséquent, seules les personnes concernées par l'application d'un système normatif pourraient participer à la délibération. Ainsi, le fonctionnement d'un système normatif pourrait-il être défini par les justiciables auquel il s'impose et pour lequel il est instauré. Dans cette visée, les tiers affecté de façon indirecte par une norme n'auraient pas qualité pour en discuté le bien fondé.

Ainsi, dans l'Église orthodoxe, l'association entre église et nation découle de l'ecclésiologie orthodoxe, considérant que la catholicité de l'Église est présente dans l'Eucharistie et donc dans les églises locales

${ }^{38}$ Jugement de la Cour suprême du Canada, Syndicat Northcrest c. Amselem, [2004] 2 R.C.S. 551, 2004 CSC 47.

39 „La religion, un reflexe identitaire. Entretien avec Odon Vallet”. Propos recueillis par Chloé Rébillard, Sciences Humaines 277/1 (2016): 40.

${ }^{40}$ Sur les origines métaphysiques du droit: Hanna Arendt, La crise de la culture. Huit exercices de pensée politique (Paris: Gallimard, 1989), 383.

${ }^{41}$ Claude Levi Strauss, Tristes tropiques (Paris: Pocket, 2001), 504.

${ }^{42}$ Marie-Hélène Popelard (ed.), Art, éducation et politique (Paris: Éditions du Sandre, 2011). La conscience politique, n'est pas le pouvoir et la domination des ego mais ,l'indépendance de la pensée, l'émancipation de l'individu, le souci publiporté à la communauté des hommes face aux chimères hystériques de la soi-disant identité nationale". 
et territoriales ${ }^{43}$, mais surtout des circonstances politiques ayant favorisé une association des églises territoriales et des peuples, leur conférant un caractère national. Dans la Russie de Pierre le grand, le spirituel s'est ainsi trouvé soumis au temporel ce qui n'est pas sans rappeler le gallicanisme en France ${ }^{44}$. Dans la Grèce actuelle, les patriarches et synodes peuvent avoir une grande influence sur le pouvoir politique. La culture byzantine promeut une conscience communautaire très affirmée, la spiritualité faisant partie intégrante de la culture commune.

Une fois établi la possible limitation de la liberté de conscience comme droit politique au sein d'une religion, il s'agit d'établir si la religion peu entrainer une limitation de la liberté de conscience comme droit civil.

\section{B. LA LIMITATION PAR LA RELIGION DE LA LIBERTÉ}

DE CONSCIENCE COMME DROIT CIVIL

Une limitation de la liberté de conscience comme droit civil pourrait découler de la limitation de la délibération des valeurs religieuses, limitation politique de la liberté de conscience. La limitation du nombre d'acteurs habilité à participer à la délibération religieuse peut en effet contribuer à une limitation des intérêts mis en lumière lors d'une délibération religieuse. Par ailleurs, il apparaît probable que la délibération étant ainsi restreinte, la nécessité pour la religion de prôner un encadrement des pratiques lui étant contraire pour subsister prenne le pas sur l'opportunité de défendre les droits de chacun dans la sphère publique.

La religion pour perdurer, peut en effet prôner un encadrement des comportements ou, au contraire, montrer le caractère obligatoire de certaines pratiques et ainsi restreindre la liberté de conscience. Ainsi la religion est-elle souvent conçue par l'opinion populaire comme limitant

${ }^{43}$ C'est à dire dans les paroisses et diocèses. L'unité est considérée comme étant assurée par la conciliarité et non par une juridiction unipersonnelle.

${ }^{44}$ Cette doctrine française, institué lors de le désaccord entre Philippe Lebel et Boniface VIII tendait à préserver le pouvoir temporel des souverains, limitant l'autorité papale. 
la liberté de conscience ${ }^{45}$. La religion peut être utilisée pour justifier la restriction d'autres religions mais aussi pour légitimer l'encadrement de la liberté de conscience. La foi en une valeur peut inciter à vouloir l'imposer pour le bien de tous en limitant la liberté de conscience de ceux qui n'y adhèrent pas. D'un point de vue religieux, la liberté peut se définir comme „la capacité de l'homme à choisir le bien" 46 . La notion de bien étant identifiée par référence à une transcende, la liberté de conscience ne se limiterait pas à adopter l'état d'esprit de son choix. Par conséquent, l'encadrement des moeurs, ne constitue pas automatiquement, d'un point de vue religieux, une limitation de la conscience. Au contraire, une limitation des actions humaines peut apparaître comme nécessaire à la préservation de la liberté de conscience de chacun.

La religion justifierait ainsi la limitation de tout comportement jugé contraire à ses exigences. S'opposant au scepticisme moral ${ }^{47}$, elle aspirerait à imposer certains comportements définis comme bons ${ }^{48}$, relevant ainsi d'une forme de paternalisme ou de perfectionnisme. La religion justifierait un ,perfectionnisme” ou paternalisme ${ }^{49}$, imposant une substitution de valeurs considérées comme absolues aux valeurs de chacun, ce qui apparaitrait difficilement admissible dans des sociétés modernes libérales, plurielles ${ }^{50}$. Elle limiterait ainsi toute expression considérée comme offensante ${ }^{51}$.

Les exemples historiques de restrictions de la liberté de conscience pour des motifs religieux abondent, le phénomène se poursuit. Nous en citerons ici quelques illustrations particulièrement évocatrices datant de $2010^{52}$.

${ }^{45}$ C'est ce qui ressort en particulier de commentaires de l'affaire „Charlie Hebdo”.

${ }^{46}$ Voir Saint Augustin, La Cité de Dieu, tome 1: Livres I à X (Paris: Points, 2004).

${ }^{47}$ Le scepticisme moral considère que les valeurs, si tant est qu'elles existent, ne sont pas accessibles à la connaissance.

${ }^{48}$ Gaudreault-Desbiens, ,Religion”, 53-66.

49 „Le paternalisme est une attitude du pouvoir, à la fois bienveillante et autoritaire, qui consiste à imposer une domination sous couvert de protection désintéressée".

${ }^{50}$ José Woehrling, „Quelle place pour la religion dans les institutions publiques?”, Le droit, la religion et le „raisonnable” (Montréal: Éditions Thémis, 2009).

${ }^{51}$ Des Versets sataniques aux caricatures danoises de Mahomet en passant par La Dernière Tentation du Christ

${ }^{52}$ Afrique: Liberté religieuse - Résumé des rapports sur certains pays - Extrait du Rapport sur la liberté religieuse dans le monde en 2010. 
En Afghanistan, les groupes minoritaires non musulmans, notamment les chrétiens, les hindous, les sikhs et les bahaïs, représentant, cumulés, près de $1 \%$ de la population, faisant l'objet de discrimination et de persécutions, tout comme la communauté minoritaire chiite. Les chrétiens étaient menacés par des arrestations et de la propagande télévisuelle. La foi bahaïe était déclarée blasphématoire par les autorités. Les populations sikhe et hindoue locales continuaient à subir diverses formes de harcèlement limitant la pratique de leur religion. En Arabie Saoudite, une police des moeurs sévi et aucune autre religion que l'islam ne peut être pratiquée. L'islam lui-même n'est pas librement pratiqué. Les chiites font l'objet d'arrestations arbitraires. Les rassemblements religieux autres que musulmans étaient ainsi interdits et les objets non musulmans confisqués. En Birmanie, le régime promouvait le bouddhisme theravāda de préférence aux autres écoles et aux autres religions, notamment dans les minorités ethniques. Le gouvernement avait, par exemple, continué de refuser de reconnaître les membres de la minorité musulmane rohingya comme citoyens, bon nombre de droits civils, économiques et sociaux leur sont refusés. En Chine, n'étaient sont autorisé que les groupes religieux affiliés aux cinq ,associations religieuses patriotiques" (bouddhistes, taoïstes, musulmans, catholiques et protestants). La répression au Tibet continuait. En Corée du nord, des services religieux seraient mis en scène pour le compte du gouvernement. La liste des pays ou la liberté de conscience a été ou est toujours limitée n'est pas exhaustive ${ }^{53}$.

Ces limitations s'accompagnent souvent des restrictions des droits de l'homme en général. Ainsi l'Arabie Saoudite a-t-elle refusé d'adhérer à la Déclaration des Droits de l'Homme et du Citoyen pour des motifs religieux ${ }^{54}$. La jurisprudence confirme qu'en apparence, nombre

${ }^{53}$ La Commission indépendante sur la liberté religieuse a ainsi relevé comme problématique la situation de nombreux pays: l'Égypte, l'Erythrée, l'Iran, l'Irak, l'Ouzbékistan, le Nigéria, le Pakistan, la Russie, le Soudan (PPP), le Tadjikistan, le Turkménistan, le Venezuela, le Vietnam.

${ }^{54}$ Abderrazac Sayadi, „L'islam face à la liberté de conscience”, Études. Revue de culture contemporaine, 5 (2011), 643-654. „Nous avons là les trois objections majeures à la notion de droits de l'homme: supériorité des droits sociaux sur les droits politiques, supériorité de l'homme blanc sur l'homme de couleur, et supériorité de la loi de Dieu sur la loi des hommes. La troisième motivation, représentée par la position saoudienne, est religieuse. Elle considère que le droit divin est supérieur aux droits de l'homme, et que la loi de Dieu, telle qu'elle est fixée par les Ulémas, ou docteurs de la Loi, dans la Shari'a, qui est la loi 
de violations de la liberté religieuse seraient causées par la religion elle-même. Nous citerons en exemples trois affaires portées devant la Cour Européenne des Droits de l'Homme: 1'affaire Buscarini et autres c. Saint-Marin, l'affaire Dimitras et autres c. Grèce et l'affaire Valsamis c.Grèce. Dans l'affaire Buscarini et autres c. Saint-Marin ${ }^{55}$, la Cour a dû rechercher si l'obligation pour des députés de prêter serment sur les Évangiles afin de pouvoir exercer leur fonction contrevenait à la Convention EDH. La Cour a conclu à une violation de l'article 9. En effet, les élus devaient par ce serment prêter allégeance à une religion. Posant une problématique similaire, l'affaire Dimitras et autres c. Grèce $e^{56}$, a permis à la Cour de constater une violation de l'article 9 de la Convention du fait de l'obligation faite aux requérants de révéler leurs convictions religieuses pour être exempts de l'obligation de prêter serment sur les Évangiles avant de pouvoir témoigner en justice.

L'imposition, par l'État, de certaines pratiques liées à la religion ou contraires à une religion, sanctionnées dans Valsamis c. Grèce $e^{57}$, est perçue comme découlant du caractère religieux de cet État. Les requérants - une famille de Témoins de Jéhovah - se plaignaient que leur troisième enfant, élève d'un lycée public, avait été sanctionnée pour avoir refusé de participer à la célébration de la fête nationale commémorant la déclaration de guerre par l'Italie fasciste à la Grèce. Les requérants, dont les convictions religieuses interdisent de s'associer à la commémoration d'une guerre, alléguaient une violation des articles 9 de la Convention (dans le chef de la fille elle-même) et 2 du Protocole $n^{\circ} 1$ (dans le chef des parents). La cour a considéré qu'une telle violation n'était pas établie ${ }^{58}$.

religieuse, est supérieure à la loi des hommes. Les droits de l'homme ne sauraient être tolérés que dans la mesure où ils sont strictement soumis aux lois religieuses de l'islam".

${ }^{55}$ Arrêt de la Cour européenne des Droits de 1'Homme [Grand Chamber], 18 fevrier 1999, affaire Buscarini et autres c. Saint-Marin (requête no24645/94).

${ }^{56}$ Arrêt de la Cour européenne des Droits de 1'Homme, 3 juin 2010, affaire Dimitras et autres c. Grèce (requêtes nos 42837/06, 3237/07, 3269/07, 35793/07, 6099/08).

${ }^{57}$ Arrêt de la Cour européenne des Droits de l'Homme, 18 décembre 1996, affaire Valsamis c. Grèce (requête no 21787/93). Recueil des arrêts et décisions 1996-VI.

${ }^{58}$ Valsamis c. Grèce, Petites Affiches, no 64 (1997): 33-37. La Cour relève d'emblée que Mlle Valsamis a été dispensée de l'enseignement religieux et de la messe orthodoxe comme elle le sollicitait en faisant état de ses propres convictions religieuses. Elle a déjà jugé, aux paragraphes 31 à 33 ci-dessus, que l'obligation de participer au défilé scolaire n'était pas de 
Il existe une contradiction conjoncturelle entre religion et liberté de conscience. Cela ne suffit en rien à démontrer la nécessité de cette opposition et ne présage nullement du rapport ontologique entre ces deux notions.

\section{LA RECONNAISSANCE PAR L'ESSENCE DE LA RELIGION DE LA LIBERTÉ DE CONSCIENCE}

La religion peut promouvoir la liberté de conscience, à la fois comme droit politique (A) et comme droit civil (B).

\section{A. LA RECONNAISSANCE PAR L'ESSENCE DE LA RELIGION DE LA LIBERTÉ DE CONSCIENCE COMME DROIT POLITIQUE}

La capacité de la religion à perdurer dans la société moderne où il n'existe plus de communauté de convictions autre qu'interstitielles montre son habilité à s'accommoder d'une norme sociale prenant son origine à l'extérieur de la société. En effet, si la religion ne reposait que sur l'homogénéité idéologique au sein d'un groupe, elle se trouverait remise en cause par l'accroissement de divergences au sein de ce groupe.

$\mathrm{Au}$ delà de la preuve historique d'une possible coïncidence entre religion et liberté de conscience, il appert que la conception de l'homme véhiculée par la religion exige le respect de sa liberté de conscience. En cela, elle ne contredit pas les idéaux démocratiques.

Nous observerons les similitudes entre les épistémologies religieuses et la connaissance du droit démocratique. La religion, si elle ne repose pas directement sur la volonté générale, source de droit démocratique, s'en accommode dans la mesure où elle n'exclut pas un élargissement du champ de la délibération publique dans la sphère politique ${ }^{59}$.

nature à heurter les convictions religieuses des parents de l'intéressée. La mesure contestée n'a pas davantage constitué une ingérence dans son droit à la liberté de religion (...). Elle considère au paragraphe 38 qu'il n'y a pas eu violation de l'article 9 de la Convention.

${ }^{59}$ Le concept hégelien de sens de 1'histoire pourrait d'ailleurs être influencé par une perception à la fois religieuse et démocratique de la société. Georg Wilhelm Friedrich Hegel, La phénoménologie de l'esprit (Paris: Gallimard, 1993). 
Une religion reposant sur un fondement traditionnel peut s'intégrer dans une société libérale si elle accepte la séparation de l'État et du culte. Ainsi l'Église catholique, si elle limite la liberté de conscience dans la recherche interne de la vérité, la défend-elle sur un plan externe ${ }^{60}$. L'absence de nécessité pour la religion de susciter l'acceptation du fait de son caractère apolitique pourrait expliquer sa capacité à reposer sur une source traditionnelle dans une société moderne plurielle. L'adhésion des fidèles n'implique pas leur insertion dans le processus de découverte. Si la religion peut être utilisée comme moyen de légitimation du pouvoir, son essence n'en demeure pas moins distincte de celle du pouvoir politique. Par conséquent, à la différence de ce dernier, elle n'a pas pour objectif principal le maintien de l'ordre public. Dans la mesure où elle peut exister sans être admise par l'ensemble de la population d'une société donnée, elle ne nécessite pas de façon absolue une adéquation entre les moeurs de cette société et les usages qu'elle entend promouvoir. Elle permet le rassemblement de ses fidèles mais ne vise pas toujours à celui de l'ensemble d'une société.

L'intégration de la question religieuse au paradigme délibératif n'apparaît pas nécessaire mais demeure possible, comme en témoigne le modèle de société ,post-séculière” établi par Habermas, modèle caractérisé par un engagement réciproque des citoyens sur la base même de leurs convictions religieuses singulières. Les convictions religieuses deviennent le fondement de l'engagement public ${ }^{61}$.

Le système démocratique peut reposer sur différentes méthodes d'identification de la volonté nationale et par conséquent encadrer la liberté religieuse de diverses façons. Les conceptions „républicaines” ou „communautaire" du fait religieux montrent que la démocratie n'impose pas une unique conception de la liberté de religion. Le point de vue religieux, lorsqu'il est admis comme source normative en démocratie, est réceptionné par le pouvoir politique à partir d'une recon-

${ }^{60}$ Voir à ce sujet Rik Torfs, „Le catholicisme et la liberté de conscience”. In: La liberté de conscience dans le champ de la religion, eds. Patrice Meyer-Bisch, Jean-Bernard Marie (Strasbourg: Documents de travail de l'IIEDH No 4, 2002), 42-50.

${ }^{61}$ Jürgen Habermas, Entre naturalisme et religion. Les défis de la démocratie, traduit de l'allemand par Christian Bouchindhomme et Alexandre Dupeyrix (Paris: Gallimard, 2008), 380 . 
naissance préalable de la légitimité ${ }^{62}$ du pluralisme ${ }^{63}$. Le dialogue étant désormais admis comme intégrant des acteurs différents, la légitimité démocratique de l'institution est désormais confortée par un dialogue interreligieux. Une fois admise la possibilité pour la religion de prendre place dans une société moderne ${ }^{64}$, il reste à montrer qu'une identification traditionnelle de la norme n'exclue pas tout processus délibératif.

Nous prendrons pour exemple diverses doctrines chrétiennes. Le protestantisme repose sur une volonté de recherche libre de la vérité religieuse et est historiquement justifié comme tel ${ }^{65}$, il n'est pas surprenant de voir la doctrine protestante prôner la défense politique d'une telle liberté ${ }^{66}$. L'influence des clercs n'est apparue que dans les débuts de l'élaboration de la théologie des débuts de l'Eglise protestante. L'édit de Nantes semble avoir induit une évolution. L'esprit des lumières semble, par la suite, avoir entrainé une méfiance envers toute tradition trop prégnante. Toute définition trop juridiquement marquée des structures de la vie ecclésiale et des frontières de l'Église est à bannir ${ }^{67}$.

La foi catholique apparaît davantage tournée vers une recherche de vérité encadrée ${ }^{68}$. Alors que le débat théologique interne à l'institution

${ }^{62}$ Est légitime ce qui est fondé en droit. En démocratie, est légitime ce qui est conforme à la volonté générale.

${ }^{63}$ Sur la multiplicité des modes de reconnaissance de l'existence de courants d'opinions religieuses divers en démocratie, voir Jean Leca, „La démocratie à l'épreuve des pluralismes", Revue française de science politique $\mathrm{n}^{\circ} 2$ (1996): 225-279.

${ }^{64}$ La modernité étant ici conçu comme l'admission de la raison comme norme transcendantale de la société.

${ }^{65}$ Gilbert Vincent, „Liberté de conscience, tolérance et protestantisme”. In: La liberté de conscience dans le champ de la religion, eds. Patrice Meyer-Bisch, Jean-Bernard Marie (Strasbourg: Documents de travail de l'IIEDH No 4, 2002), 56.

${ }^{66}$ Le constitutionnalisme nord-américaine aurait été influencé par une éthique protestante. Voir, à ce sujet, Fernando Rey Martínez, La ética protestante y el espiritu del constitucionalismo (Bogotá: Universidad, Externado de Colombia, 2003).

${ }^{67}$ Michael Walzer, La révolution des saints, éthique protestante et radicalisme politique (Paris: Belin, 1987).

${ }^{68}$ Sur la délibération dans l'Eglise catholique: Guillaume Desprez, Jacques Garrigan, „Tome 1: De la foi catholique \& de la doctrine de l'Eglise”. In: Recueil des actes, titres et mémoires concernant les affaires du clergé de France, augmenté d'un grand nombre de pièces $\&$ dobservations sur la discipline présente de l'Eglise. Divisé en douze tomes, et mis en nouvel ordre, suivant la délibération de l'Assemblée générale du Clergé. Du 29. Avril 1705 (Avignon: chez Jacques Garrigan, M. DCC. LXVIII, 1768-1780). 
privilégie la recherche de vérité sur la liberté de conscience, l'Église ne promeut pas une telle hiérarchie à l'externe, elle semble encourager une primauté de la liberté de conscience dans le débat public. La pensée catholique ne refuse pas tout débat sur une vérité admise. La délibération semble en effet, au delà du dogme, pour tout point non encore établi, demeurer source de connaissance, conformément à la rhétorique de la modernité. En d'autres termes, la tradition reste en marche. Une Vérité universelle pourrait coexister avec une vérité locale, des habitudes d'action contextualisée, ne s'éprouvant qu'aux intérêts qu'elle satisfait. La pensée catholique rencontre la philosophie post-moderne ${ }^{69}$ par son approche pragmatique de la vérité et l'admission de l'expérience dans la découverte de la vérité en particulier.

Qui plus est, une conception naturaliste de $1^{\text {'homme }}$, $^{70}$ assumant l'existence d'une nature humaine ${ }^{71}$ s'imposant comme source de droit indépendamment de la volonté humaine, n'exclurait pas nécessairement l'admission de la légitimité de la délibération publique, constituant un moyen d'identification d'une telle nature. En admettant que la vérité ne puisse être conçue indépendamment de la liberté, le concile Vatican II marque un trait d'union entre une approche subjective et moderne de la liberté et une approche traditionnelle reconnaissant l'union ontologique entre la personne et la vérité. Le caractère intégral de la foi n'implique pas nécessairement le refus de la sécularisation ${ }^{72}$. Le concile Vatican II a su trouver un compromis entre la protection de la vérité et la liberté de conscience ${ }^{73}$. Quoique la vérité existe et qu'il faille y adhérer, rien

${ }^{69}$ Gilles Deleuze, Empirisme et subjectivité (Paris: PUF, 1953), 160.

${ }^{70}$ Une conception naturaliste de l'homme peut en particulier considérer la religion comme nécessaire au développement humain.

${ }^{71}$ John Dewey, ,Démocratie et nature humaine”, Revue du MAUSS n 19/1 (2002): 113-126. DOI: 10.3917/rdm.019.0113. URL: https://www.cairn.info/revue-du-mauss-20021-page-113.htm

${ }^{72}$ Cf. M. Diet, „Église et État selon Vatican II”, Ephemerides Theologicae Lovanienses 1978, 152. Cf. aussi la dissertation doctorale du même auteur, M. Diet, Kerk en Staat volgens het Tweede Vaticaanse Concilie: de godsdienstvrijheidalsgrondslagvanhunonderlingeverhouding (Leuven, s.n., 1977, xxxvi+ 197).

${ }^{73}, \ldots .$. la vérité doit être cherchée selon la manière propre à la personne humaine et à sa nature sociale, à savoir par une libre recherche, par le moyen de l'enseignement ou l'éducation, de l'échange et du dialogue par lesquels les uns exposent aux autres la vérité qu'ils ont 
ne justifie que cette adhésion soit contrainte par le droit. La majorité au concile a reconnu que la dignité ne pouvait se concevoir sans reconnaissance par le droit de la liberté de chacun.

Ces exemples semblent indiquer que la limitation de la liberté de conscience ne découle pas de la religion en elle-même. Elle pourrait résulter de son utilisation politique, destinée à légitimer des revendications identitaires. Ce type d'instrumentalisation de la religion devrait se raréfier dans la société moderne où la religion repose sur un principe autonome. Ce type de limitation de la liberté de conscience n'apparaît pas en conséquence comme une spécificité de la religion Nombre de limitations de l'expression religieuse ne découlent pas tant de la religion elle-même, qui pour assurer sa survie admet la liberté d'expression de toutes croyances, que d'une conception stricte de la laïcité, considérant l'expression religieuse comme aboutissant à une limitation systématique de la liberté Aussi existe-t-il existe un grand nombre de systèmes totalitaires dénués de toutes sources religieuses: nazisme, bolchevisme ${ }^{74}$.Elle n'est possible qu'en raison des limites pratiques de mise en œuvre du système démocratique $^{75}$. Ces difficultés résultent en particulier des entraves à une pleine circulation de l'information et à sa réception par les citoyens. Ce risque d'erreur est accru par la conquête du pouvoir par la classe politique, l'utilisation de sondages d'opinion ou d'études sur le comportement de l'électorat contre les intérêts de ce dernier. Les représentants manquent à leurs engagements sous prétexte de s'adapter aux circonstances.

La religion n'apparaît donc pas comme un obstacle à la protection de la liberté comme droit politique, reste à déterminer si elle peut justifier la limitation de la liberté de conscience comme droit civil. Il existe divers moyens de concevoir la liberté de conscience ${ }^{76}$, concep-

trouvée ou pensent avoir trouvée, afin de s'aider mutuellement dans la quête de la vérité." Concile ocuménique Vatican II, Déclaration sur la liberté religieuse Dignitatis Humanae, In: Documents Conciliaires III, L'Église dans le monde. L'apostolat des laïcs. La liberté religieuse. Les moyens de communication sociale (Paris: Centurion n³, 1966), 354-356.

${ }^{74}$ Le totalitarisme était pour Raymon Aaron une ,religion séculière”. Raymond Aaron, L'opium des intellectuels (Paris: Pluriel, 2010).

75 „La démocratie est le pire des régimes à l'exception de tous les autres” W. Churchill.

${ }^{76}$ La définition de la liberté de conscience dépend en particulier du processus de découverte normative privilégié par un système de droit. 
tion subjective si l'on se place du point de vue de celui qui l'exerce ou objective si l'on se réfère à des croyances „officielles”

„Il existe une dichotomie entre l'approche subjective, où l'on évalue la pratique en fonction des croyances sincères de l'individu indépendamment de leur correspondance avec une quelconque théologie reconnue comme officielle, et l'approche objective, où la protection juridique accordée à la pratique alléguée comme religieuse est fonction de sa correspondance à la théologie officielle de la foi à laquelle l'individu adhère" 77 .

\section{B. LA RECONNAISSANCE PAR L'ESSENCE DE LA RELIGION DE LA LIBERTÉ}

DE CONSCIENCE COMME DROIT CIVIL

La protection de la liberté de conscience par la religion semble constituer davantage qu'une simple possibilité historique; le concept de liberté de conscience pourrait se trouver enraciné dans la religion ${ }^{78}$. La limitation de la liberté de religion découle davantage d'une conception paternaliste de la laïcité consistant à voir dans la religion une aliénation systématique que de la religion elle-même, ayant intérêt à assurer le respect de la liberté de conscience pour se préserver.

Il suffit d'observer la plupart des États assurant la protection de la liberté de conscience pour observer que la religion y perdure, démontrant la possible coexistence de la religion et de la liberté de conscience. Si tant est qu'un État dont la population serait globalement athée puisse exister, il semble difficilement envisageable qu'une population entière soit athée sans aucune limitation politique de la liberté de conscience. Ainsi, la limitation de la liberté de la conscience par la religion, aussi

${ }^{77}$ Jean-François Gaudreault-Desbiens, „Religion, expression et libertés: l'offense comme raison faible de la régulation juridique", Cahiers de la Recherche sur les Droits Fondamentaux n 8 (2010): 53-66.

${ }^{78}$ Sur la possibilité historique d'une défense de la liberté religieuse par la religion: Sollicitant l'aide de Louis IX contre l'empereur, le pape Grégoire IX lui écrit en 1239: „Dieu choisit la France de préférence à toutes les autres nations de la terre, pour la protection de la foi catholique et pour la défense de la liberté religieuse. Pour ce motif, la France est le Royaume de Dieu même, les ennemis de la France sont les ennemis du Christ". 
choquante soit-elle, apparaît comme une exception dans les systèmes démocratiques.

Il reste à déterminer si un État religieux peut assurer la liberté de conscience. La religion ne recommande pas systématiquement la limitation de la liberté de conscience, même si elle peut être détournée à cette fin. La pensée chrétienne, par exemple, prévoit la possibilité pour chacun de faire de la religion une affaire privée ${ }^{79}$. Nombre de concepts ayant influencé le monde politique moderne s'ancrent dans la théologie. Ainsi en est-il de la laïcité, de la liberté, de l'égalité, du bien commun, du caractère privé de la religion, de la valeur de la personne, ou de la volontée ${ }^{80}$.

Dans la religion chrétienne, l'Évangile rappelle la séparation de l'ordre politique ${ }^{81}$ et de l'ordre religieux ${ }^{82}$ et le respect du pouvoir politique en place. La spiritualité y est présentée comme relevant du for intérieur ${ }^{83}$. La doctrine catholique, en particulier, prétend à l'universalité. L'histoire n'a cessé de cantonner ces présentons au pouvoir tempo$\mathrm{rel}^{84}$. L'encyclique Vatican II admet explicitement le droit de toutes les religions à une expression publique en démocratie. L'origine biblique de la laïcité ${ }^{85}$ est cependant controversée. Certains penseurs, tels que Lucien Febvre considèrent que la laïcité ne se constitue qu'en s'émancipant du divin ${ }^{86}$.

79 „Le christianisme est la religion de la sortie de la religion”. Marcel Gauchet, Un monde désenchanté (Paris: Les éditions de l'atelier, 2004), 253.

${ }^{80}$ A titre d'exemple sur l'égalité: „Ce que vous ferez au plus petit d'entre les miens, c'est à moi que vous le ferez", Evangile de Jésus-Christ selon saint Matthieu 18:1-5.10.12-14.

${ }^{81}$, „Il n'y a plus ni Juif ni Grec, il n'y a plus ni esclave ni libre, il n'y a plus ni homme ni femme; car tous vous êtes un en Jésus-Christ', Galates 3:28.

${ }^{82}$ „Rendez à César ce qui est à César et à Dieu ce qui est à Dieu!”, Luc 20:25; ,Mon royaume n'est pas de ce monde, répondit Jésus”, Jean 18:36.

${ }^{83}$ „Ce que vous faites pour devenir des justes, évitez de l'accomplir devant les hommes pour vous faire remarquer. Sinon, il n'y a pas de récompense pour vous auprès de votre Père qui est aux cieux." Evangile de Jésus-Christ selon saint Matthieu (6:1-6.16-18).

${ }^{84}$ Sur la consécration historique du divorce entre pouvoir temporel et religion chrétienne, voir Julien Souplet, „Histoire des idées politiques en occident”, L'éléphant 7 (2014): 72.

${ }^{85}$ Mollat Guillaume, Georges de Lagarde, „La naissance de l'esprit laïque au déclin du Moyen-Âge, t. I. Bilan du XIIIe siècle; t. II. Marsile de Padoue ou le premier théoricien de l'État laïque". In: Revue des Sciences Religieuses 15/2 (1935): 307-309.

${ }^{86}$ Alphonse de Châteaubriant, dans un ouvrage posthume, Itinerarrium ad lumen divinum (1953), a estimé qu'il fallait remonter jusqu'au thomisme, qui rompt avec la pensée 
Les assimilations „nation-religion” dans l'Eglise orthodoxe ont été condamnées par le Concile de Constantinople en 1872 sous le nom de phylétisme (,phylé”, la tribu), même si cette condamnation est demeurée sans grande portée. La complémentarité byzantine entre spirituel et temporel ne vise pas, à l'origine, à une prépondérance du temporel sur le spirituel. Il ne s'agit nullement de limiter la liberté de conscience sur un plan personnel ${ }^{87}$. La liberté de conscience, en effet, non seulement a, historiquement, été assurée dans des États religieux mais est recommandée par des textes religieux. Il semblerait qu'une religion d'État, motivée par des raisons culturelles entre autres, n'implique pas une limitation de la liberté de conscience. L'idée même de religion impliquerait le respect de la liberté de conscience, et une forme de laïcité, suivant le sens donné à ce dernier terme ${ }^{88}$.

Sur un plan plus théorique, le principe même de religion suppose une liberté de conscience. Si l'Homme, dans son état naturel, possède le libre arbitre, un pouvoir humain apparaît peu légitimement à le restreindre. La plupart des religions reconnaissent l'égale valeur de chaque conscience ${ }^{89}$. La vérité est accessible à tous ${ }^{90}$. De l'égalité des consciences, découle tout naturellement l'égal droit de chacune d'elle à s'épanouir. La démocratie partage cette perspective. Un système connaissant le peuple comme moyen de gouvernement ne se contente pas de reconnaître la valeur de l'homme mais aussi la valeur suprême de la conscience. Ce principe justifie celui d'égalité devant la loi et le rejet de la domination de certains hommes sur d'autres.

symbolique des Pères (dont Bonaventure sera le dernier grand représentant) en substituant l'aristotélisme au platonisme dans la théologie catholique.

${ }^{87}$ Concile pan-orthodoxe (Constantinople, 1872). Nominalisme de Marsile de Padoue et de Guillaume d'Ockham.

${ }^{88}$ Catherine Kintzler, Penser la laïcité (Paris: Minerve, 2014).

${ }^{89}$ „Ce que vous ferez au plus petit d'entre les miens, c'est à moi que vous le ferez”. Evangile de Jésus-Christ selon saint Matthieu (25:40).

${ }^{90} \mathrm{La}$ foi chrétienne, par exemple, considère que Dieu a placé dans le cœur de tout homme une intelligence. „Maintenant donc, ces trois-là demeurent, la foi (pistis), l'espérance (helpis) et l'amour (ou: charité, agapè) mais l'amour est le plus grand" Paul (I Co 13:13). Dieu créa l'homme à son image, à l'image de Dieu il le créa (La Genèse). De plus, l'espérance constitue une vertu théologale. 
La reconnaissance d'une adéquation entre la démocratie comme gouvernement du peuple et la démocratie comme gouvernement pour le peuple suppose un certain scepticisme moral ${ }^{91}$. En effet, si l'on considère que, comme semble l'attester le désaccord entre les hommes, il n'existe aucun moyen d'identifier les valeurs en adéquation avec l'intérêt général, la référence à la volonté générale demeure le meilleur moyen de s'en approcher. La religion, à l'inverse, supposerait l'existence mais aussi la connaissance d'une loi, transcendant la volonté humaine. La contradiction n'est qu'apparente. En effet, cette loi n'est pas acceptée par tous. Il semble dès lors malaisé de l'imposer à ceux qui ne l'ont pas encore reçue. Privilégier de façon définitive un système de valeur relève d'un certain dogmatisme et implique une hiérarchie entre les consciences et par conséquent entre les hommes.

D'un point de vue religieux, la négation du libre arbitre de celui à qui s'impose une norme religieuse pourrait s'avérer un tort plus grave encore que l'infraction de cette norme, à condition toutefois que la norme religieuse en question ne vise pas à préserver une conscience ou son siège. La norme religieuse n'a de vertu qu'une fois comprise et donc admise. Si l'on considère la conscience comme la caractéristique essentielle du phénomène humain, la liberté de conscience constitue la première des libertés, le seul moyen pour l'homme de s'émanciper. Épistémologie religieuse et épistémologie du droit démocratique se rejoignent en cela.

En effet, en démocratie, les normes ne peuvent être dégagées selon un critère objectif, par la référence à d'autres valeurs, puisqu'aucune valeur, aucun dogme, ne s'impose à tous. Par conséquent, la démocratie repose sur une foi en la capacité de l'homme à identifier ce qui est bon pour lui. Elle reconnaît la capacité de la conscience de chacun à percevoir une part de la vérité et à se compléter dans sa recherche. Cette conviction tient lieu de pacte social. L'exercice du pouvoir par tous constitue le plus sûr moyen d'assurer la défense des droits de chacun. Une réflexion commune prend en compte un grand nombre d'informations.

${ }^{91}$ Sandra Laugier, „Wittgenstein: politique du scepticisme”, Cités 38/2 (2009): 109-127. 
Un système de réflexion en réseau, fondé sur un échange de données entre plusieurs groupes, en favorisant une meilleur diffusion de la connaissance, permet une description plus complexe et donc plus exacte des exigences de chacun ${ }^{92}$. La participation de chacun à la vie politique, une certaine mutualité des relations entre gouvernant et gouverné, caractérise donc la démocratie autant que la protection des droits fondamentaux des individus.

La démocratie ne peut être définie de façon purement substantielle, par les droits qu'elle préserve. En effet, de nombreux régimes non démocratiques prétendent protéger l'homme et organisent la protection de droits fondamentaux par des chartes ou constitutions. Démocratie délibérative et démocratie substantielle ne peuvent être conciliés que si le citoyen est socialisé à rechercher l'intérêt collectif ${ }^{93}$. Ces deux aspects de la démocratie entreraient en tension si chaque citoyen recherche son propre avantage. Il est difficile de maximiser les droits de chacun tout en respectant au mieux la volonté de chacun ${ }^{94}$. C'est pourtant ce à quoi tend une démocratie, et, a fortiori une démocratie sociale.

La reconnaissance première de la foi en la valeur de la conscience humaine, surpassant toute autre conviction, implique le refus de l'alphabétisation d'un système de valeur. La démocratie implique que le droit doive suivre l'évolution des mentalités et non la précéder. La déclaration universelle des droits de l'homme considère que „tous les êtres humains" sont doués de raison et de conscience ${ }^{95}$. La religion s'intègre au cadre démocratique dans la mesure où elle s'accommode d'un scepticisme moral dans l'exercice du pouvoir. Imposer des valeurs ${ }^{96}$ équivaudrait à reconnaître la suprématie de la conscience de ceux qui

${ }^{92}$ Émile Gravier, „On peut tromper une fois mille personnes, mille fois une personne, mais pas mille fois mille personnes”. Un film „La Cité de la peur, une comédie familiale” réalisé par Alain Berbérian, 1994.

${ }^{93} \mathrm{Cf}$. la notion de voile d'ignorance, en particulier dans la philosophie de John Rawls. John Rawls, Théorie de la justice (Paris: Points, 2009).

${ }^{94}$ John Stuart Mill, Jérémy Bentham, Utilitarianism and Other Essays (London: Penguin, 1987).

${ }_{95}$ Déclaration universelle des droits de l'Homme du 10 décembre 1948, Art. $1^{\text {er }}$.

${ }^{96}$ A titre d'exemple, l'évangile précise que „Dieu résiste aux orgueilleux, Mais il fait grâce aux humbles” Jacques (4:6). „Ce que tu as caché aux sages et aux savants, tu l'as révélé aux tout-petits" Matthieu (11:25-27). 
les supportent et paraît en cela difficilement conciliable avec la reconnaissance de l'égalité des consciences.

Si la religion implique la reconnaissance de la liberté de conscience, la reconnaissance de la liberté de conscience suppose réciproquement un acte de foi en la conscience humaine. L'adhésion au système démocratique, fondé sur la reconnaissance de la liberté de conscience, ne peut être fondé sur un raisonnement déductif ${ }^{97}$.

\section{CONCLUSION}

Les influences de la religion sur la liberté de conscience ne peuvent être définies de façon univoque. Si la religion est souvent conçue comme limitant la liberté de conscience, l'adoption de la démocratie délibérative comme principe de gouvernement, réconciliant les deux aspects de la liberté de conscience, entendue comme droit civil et comme droit politique $^{98}$, pourrait reposer sur une conception de la personne d'origine religieuse.

La religion et la liberté de conscience apparaissent entretenir une relation d'opposition et de complémentarité. La limitation de la liberté de conscience par la religion s'expliquerait, sur un plan anthropologique, par la fonction identitaire assurée par la religion, se trouvant instrumentalisée comme une idéologie pourrait l'être. La religion peut être utilisée comme alibi pour légitimer la restriction de la liberté de conscience ${ }^{99}$ mais ne saurait l'exiger en elle-même. Les motifs théoriques justifiant une limitation de la liberté par la conscience de la

${ }^{97}$ Sur les rapports entre religion et rationalité, voir Martin Heidegger, Chemins qui ne mènent nulle part (Paris: Gallimard, 1962).

${ }^{98}$ Les droits civils et politiques étant définis visent à protéger les individus par rapport à l'Etat et les droits politiques, comme ceux garantissant aux individus de pouvoir participer à la gestion des affaires publiques.

99 „la religion polarise les sociétés libérales contemporaines serait un euphémisme: elle fait plus que jamais office de variable ,paratonnerre” sur laquelle se concentrent de nombreux autres débats qui n'ont souvent qu'un lien bien ténu avec elle: identité politique, terrorisme, immigration, etc.”. Jean-François Gaudreault-Desbiens, ,Religion, expression et libertés: l'offense comme raison faible de la régulation juridique", Cahiers de la Recherche sur les Droits Fondamentaux 8 (2010): 53-66. 
religion ne seraient que des rationalisations postérieures d'une évolution résultant de causes purement pratiques. Au contraire, l'essence de la religion pourrait amener l'homme à dépasser une identité culturelle pour tendre vers ce qui le transcende. La délibération entre individus distincts, possible dans la seule hypothèse ou la liberté de conscience est assurée, devrait permettre cela.

En outre, la religion, apolitique, peut subsister sans être acceptée de tous. Son fondement n'est pas contractuel. Par conséquent, elle est en mesure de conserver une source traditionnelle dans une société plurielle. La tradition n'exclut pas nécessairement une forme de dialogue. Au-delà de la preuve d'une possible coexistence de la religion et du pluralisme politique, la religion implique, par essence, la reconnaissance de la liberté de conscience. L'admission par la religion d'une reconnaissance de la liberté de conscience découle du libre arbitre ${ }^{100}$ résultant de la condition humaine même ${ }^{101}$.

En retour, la liberté de conscience repose sur une conception de l'être humain s'appuyant sur la foi en l'égale valeur de chaque conscience, ce qui relève d'une démarche similaire à celle de la religion. Ainsi la conscience est-elle reconnue non seulement comme le moyen mais aussi la finalité du pouvoir politique. Si la reconnaissance de la liberté de conscience et la religion découlent de fondements philosophiques communs, l'encadrement juridique de la liberté de conscience rétroagit sur la religion dont elle cerne l'exercice.

La religion constitue une source d'influence de la définition juridique de la liberté de conscience. La reconnaissance de la liberté de conscience par l'essence de la religion n'implique nullement la reconnaissance par l'État démocratique, de cette gouvernance de la religion. L'instrumentalisation politique de la religion pouvant détourner la religion de sa vocation spirituelle initiale, l'État démocratique peut se trouver dans l'obligation d'opérer un encadrement législatif du fait religieux pour assurer la protection de la liberté de conscience.

${ }^{100}$ Sur les conséquences politiques de la liberté conférée à l'homme par Dieu, voir Saint Thomas d'Aquin, Somme théologique, tome 1 (Paris: CERF, 1984).

${ }^{101}$ Arthur Schopenhauer, Essai sur le libre-arbitre (6e édition). Traduit en français pour la première fois et annoté par Salomon Reinach (Paris: F. Alcan, 1894), 176. 


\section{RÉFÉRENCES}

Aaron, Raymond. L'opium des intellectuels. Introduction par Nicolas Baverez. Paris: Pluriel, 2010.

Arendt, Hannah. Essays in Understanding: 1930-1954. Formation, Exile, and Totalitarianism, edited by Jerome Kohn, (New York: Harcourt Brace\&Co., 1994). Texte traduit par Etienne Tassin et publié dans Les Origines du totalitarisme. Eichmann à Jérusalem (Paris: Collection Quarto, Gallimard, 2002): 967-974.

Arendt, Hannah. La crise de la culture. Paris: Gallimard, 1989.

Arendt, Hannah. Le système totalitaire. Les origines du totalitarisme. Nouvelle édition. Paris: Edition Points, 2005.

Deleuze, Gilles. Empirisme et subjectivité. Paris: PUF, 1953.

Dewey, John. „Démocratie et nature humaine”. Revue du MAUSS n 9 (2002): 113-126. DOI : 10.3917/rdm.019.0113.

Draï, Raphaël. „La liberté dans la pensée juive”. Raisons politiques 8/4 (2002): 61-80.

Draï, Raphaël. La Thora. La législation de Dieu. Paris: Michalon, 2000.

Gauchet, Marcel. Un monde désenchanté. Paris: Les éditions de l'atelier, 2004. Gaudreault-Desbiens, Jean-François. „Religion, expression et libertés: l'offense comme raison faible de la régulation juridique". Cahiers de la Recherche sur les Droits Fondamentaux n 8 (2010): 53-66.

Gilbert, Vincent. „Liberté de conscience, tolérance et protestantisme”. In: La liberté de conscience dans le champ de la religion, eds. Patrice Meyer-Bisch, Jean-Bernard Marie. Strasbourg: Documents de travail de l'IIEDH No 4, 2002, 56-74.

Guillaume, Mollat, Georges de Lagarde. „La naissance de l'esprit laïque au déclin du Moyen-Âge, t. I. Bilan du XIIIe siècle; t. II. Marsile de Padoue ou le premier théoricien de l'État laïque". Revue des Sciences Religieuses 15/2 (1935): 307-309.

Habermas, Jürgen. Entre naturalisme et religion. Les défis de la démocratie. Traduit de l'allemand par Christian Bouchindhomme et Alexandre Dupeyrix. Paris: Gallimard, 2008.

Hansen, Mogens Herman. Démocratie athénienne - Démocratie moderne: tradition et influences. Vandoeuvre-Genève: Entretiens sur l'Antiquité Classique 56, 2010.

Hegel, Georg Wilhelm Friedrich. La phénoménologie de l'esprit. Paris: Gallimard, 1993. 
Heidegger, Martin. Chemins qui ne mènent nulle part. Paris: Gallimard, 1962. Kintzler, Catherine. Penser la laïcité. Paris: Broché, 2014.

Laugier, Sandra. „Wittgenstein: politique du scepticisme”. Cités 38/2 (2009): 109-127.

Leca, Jean, „La démocratie à l'épreuve des pluralismes”, Revue française de science politique $\mathrm{n}^{\circ} 2$ (1996): 225-279. DOI: 10.3406/rfsp.1996.395052

Lévi-Strauss, Claude. Tristes tropiques. Paris: Pocket, 2001.

Martínez, Fernando Rey. La ética protestante y el espiritu del constitucionalismo. Bogotá: Universidad, Externado de Colombia, 2003.

Mill, John Stuart, Bentham, Jérémy. Utilitarianism and Other Essays. London: Penguin, 1987.

Morerod, Charles. „Dogme et Oecuménisme”, Nova et Vetera 78/1-2 (2003): 29-61.

Murdoch, Jim. La protection du droit à la liberté de pensée, de conscience et de religion par la Convention européenne des droits de l'homme. Série des précis sur les droits de l'homme du Conseil de l'Europe. Strasbourg: Les ateliers du Conseil de l'Europe, 2012.

Puech, Aimé. Histoire de la littérature grecque chrétienne depuis les origines jusqu'à la fin du IVeme siècle, 3 tome. Paris: Société d'édition Les Belles Lettres, 1928-1930.

Rawls, John. Théorie de la justice. Paris: Poche, 2009.

Sartre, Jean-Paul. L'être et le néant. Paris: Gallimard, 1976.

Sayadi, Abderrazac. „L'islam face à al liberté de conscience”. Études. Revue de culture contemporaine n 5 (2011): 643-654.

Schopenhauer, Arthur. Essai sur le libre-arbitre (6e édition). Traduit en français pour la première fois et annoté par Salomon Reinach. Paris: F. Alcan, 1894. Souplet, Julien. „Histoire des idées politiques en occident”. L'éléphant n 7 (2014): 72.

Torfs, Rik. „Le catholicisme et la liberté de conscience”. In: La liberté de conscience dans le champ de la religion, eds. Patrice Meyer-Bisch, Jean-Bernard Marie. Strasbourg: Documents de travail de l'IIEDH No 4, 2002, 42-50.

Tresmontant, Claude. Introduction à la théologie chrétienne. Paris: Seuil, 1974.

Walzer, Michael. La révolution des saints - Ethique protestante et radicalisme politique. Paris: Belin, 1987.

Woehrling, José. „Quelle place pour la religion dans les institutions publiques?”. In: Le droit, la religion et le „raisonnable”. Le fait religieux entre 
monisme étatique et pluralisme juridique, ed. Jean-François Gaudreault-Desbiens. 150-164. Montréal: Éditions Thémis, 2009.

\section{ZARZĄDZANIE KWESTIAMI RELIGIJNYMI \\ I WOLNOŚCIĄ SUMIENIA}

\section{Streszczenie}

Wpływu religii na wolność sumienia nie sposób wyjaśnić za pomocą prostych schematów. Często religia jest postrzegana jako ograniczenie wolności sumienia. Z perspektywy filozofii religii należy jednak podkreślać znaczenie wyboru demokracji jako zasady ustroju. Na poziomie antropologicznym ograniczanie znaczenia wolności sumienia przez religię może być wyjaśniane $\mathrm{z}$ odwołaniem się do funkcji identyfikacyjnej realizowanej przez religię. $\mathrm{Z}$ drugiej strony, dla swego istnienia religia nie potrzebuje akceptacji ze strony wszystkich. Poza tym, że istnieją oczywiste dowody na możliwą koegzystencję religii i pluralizmu politycznego, religia zakłada w swojej istocie uznanie wolności sumienia. Poszanowanie wolności sumienia wynika z samej natury człowieka. Religia pozwala człowiekowi na przekroczenie jego tożsamości kulturowej. Natomiast wolność sumienia opiera się na przekonaniu o równej wartości sumienia każdego człowieka. Nie jest ona jedynie środkiem, ale również jednym z celów władzy politycznej. Religia i wolność sumienia mają wspólne podstawy. Prawne uznanie wolności sumienia ma pośredni wpływ na religię poprzez regulacje dotyczące korzystania z tej wolności.

Słowa kluczowe: wolność sumienia; epistemologia religii; demokracja; prawa obywatelskie; prawa polityczne; wolność sumienia i religii; wolność sumienia i wyznania

GOVERNANCE OF RELIGION AND FREEDOM OF CONSCIENCE

\section{Summary}

The influence of religion on the freedom of conscience cannot be defined in simple terms. Religion is often conceived as limiting the freedom of conscience. However, from the perspective of the philosophy of religion it is necessary 
to underline the significance of the adoption of democracy as a principle of government. The limitation of the freedom of conscience by religion could be explained, on an anthropological level, by the identity function realized by religion. Nevertheless, religion can exist without being accepted by all. Beyond the evidence of a possible coexistence of religion and political pluralism, religion inherently implies the recognition of the freedom of conscience. The respect for the freedom of conscience results from the human condition itself. Religion makes it possible to transcend one's cultural identity. In return, the freedom of conscience is based on a faith in the equal value of the conscience of every human being. The latter is recognized not only as the means but also as one of the purposes of political power. Religion and the freedom of conscience seem to derive from common foundations. The legal recognition of the freedom of conscience has a concomitant impact on religion by the regulation of its exercise.

Key words: freedom of conscience; religious epistemology; democracy; civil rights; political rights; religious freedom; freedom of conscience and religion 